\title{
Aporte al conocimiento de las medusas (Cnidaria: Medusozoa) en el departamento del Atlántico, Colombia
}

\author{
Jeferson Durán-Fuentes ${ }^{1, *}$, Adriana Gracia C. ${ }^{1}$, Carlos Osorio M. ${ }^{1}$, Cristina Cedeño-Posso ${ }^{2}$ \\ ${ }^{1}$ Programa de Biología, Facultad de Ciencias Básicas, Universidad del Atlántico, Atlántico, Colombia \\ ${ }^{2}$ Grupo de Investigaciones en Manejo y Conservación de Fauna, Flora y Ecosistemas Estratégicos Neotropicales, \\ Universidad del Magdalena, Santa Marta, Colombia
}

\begin{abstract}
Resumen
El subfilo Medusozoa (clases Cubozoa, Hydrozoa y Scyphozoa) se caracteriza por un esquema corporal predominantemente medusoide. El subfilo agrupa organismos con características pelágicas, lo que en cierta medida ha dificultado su conocimiento y estudio. En el Caribe colombiano se han registrado 25 de las 130 especies presentes en el mar Caribe, pero se considera que aún debe ahondarse en el conocimiento en torno a su diversidad local y regional. En este trabajo se registró la composición de las especies de Medusozoa presentes en algunas localidades del departamento del Atlántico, con énfasis en el sector turístico de Puerto Velero. Los registros se obtuvieron entre agosto de 2016 y diciembre de 2017. Las muestras se recolectaron de diversas formas: manualmente, utilizando arrastres superficiales con una red rectangular $(1 \mathrm{~mm}$ de apertura de malla), o durante actividades de pesca. Se observaron siete especies: una de Hydrozoa, una de Cubozoa y cinco de Scyphozoa. Aurelia aurita y Chrysaora sp. fueron las más frecuentes en el sector de Puerto Velero. Aunque la mayoría de las especies son de distribución amplia o cosmopolita y ocasionalmente llegan a las costas del Caribe colombiano, en el Atlántico solo se tenía registro de una de ellas, por lo que estas observaciones amplían la información sobre su diversidad y frecuencia de aparición en esta zona del país, lo cual es de importancia para gestión y conservación en los lugares que desarrollan actividades turísticas. (C) 2018. Acad. Colomb. Cienc. Ex. Fis. Nat.
\end{abstract}

Palabras clave: Caribe colombiano; Zona turística; Medusas.

Contribution to the knowledge on jellyfishes (Cnidaria: Medusozoa) in the department of Atlántico, Colombia

\begin{abstract}
The Medusozoa subphylum (Cubozoa, Hydrozoa, and Scyphozoa classes) is characterized mainly by a medusoid body scheme. The subphylum groups together organisms with pelagic characteristics, which to some extent has hindered their knowledge and study. Twenty five of the 130 species found in the Caribbean Sea have been recorded for the Colombian Caribbean, but new efforts are required to understand their diversity at local and regional level. In this study we registered the composition of subphyla species in some localities of the department of Atlántico, with an emphasis in the Puerto Velero tourist area. We collected the material between August 2016 and December 2017. Samples were collected manually, using a rectangular surface net (1 mm mesh opening), or during fishing activities. We recorded seven species: One from the Hydrozoa subphylum, one from the Cubozoa, and five from the Scyphozoa. Among these, Aurelia aurita and Chrysaora sp. were the most frequent in the Puerto Velero area. Although most of these species are widely distributed or cosmopolitan, and occasionally reach the Colombian Caribbean coasts, only one of them was previously recorded in the department of Atlántico. This new register increases the knowledge regarding their diversity and frequency of occurrence in this area of the country, and it is of particular interest for tourist places, especially for management and conservation purposes. (C) 2018. Acad. Colomb. Cienc. Ex. Fis. Nat.
\end{abstract}

Key words: Colombian Caribbean; Tourist area; Jellyfishes.

\section{Introducción}

El filo Cnidaria Verrill, 1865, está constituido por un grupo de invertebrados, predominantemente marino, que abarca unas 11.665 especies descritas (WoRMS, 2017), entre medusas, anémonas, corales, hidras de agua dulce y otras menos conocidas (Collins, 2002; Daly, et al., 2007). La principal característica de las especies agrupadas en este filo es la presencia de células urticantes, los cnidocitos, las cuales contienen un orgánulo denominado nematocisto
(Marques \& Collins, 2004); estas células contienen toxinas (neurotoxinas, cardiotoxinas, nefrotoxinas, miotoxinas, etc.) que funcionan como mecanismo de ataque (captura de presas) o defensa (Vera, et al., 2004; Gasca \& LomanRamos, 2014; Jouiaei, et al., 2015).

\footnotetext{
*Correspondencia:

Jeferson Durán-Fuentes, jalexisduran@mail.uniatlantico.edu.co

Recibido: 13 de septiembre de 2017

Aceptado: 1 de febrero de 2018

Editor: Marta Ramírez Pinilla
} 
En la actualidad Cnidaria se encuentra dividido en dos subfilos, Anthozoa y Medusozoa (Marques \& Collins, 2004; Technau, et al., 2015). Los antozoos se presentan solo como pólipos solitarios o coloniales, mientras que los medusozoos (clases Staurozoa, Hydrozoa, Scyphozoa y Cubozoa), además de formar pólipos solitarios o coloniales, forman una medusa que contiene gametos (Technau, et al., 2015); así mismo, exhiben un ciclo de vida característico con, por lo menos, tres fases: una larva plánula, un pólipo generalmente sésil, y una medusa generalmente pelágica (Collins, 2002; Marques \& Collins, 2004; Collins, et al., 2006).

Las medusas se caracterizan por ser uno de los organismos más importantes de las comunidades bióticas marinas del necton y del zooplancton (Boero, 2013). Debido a su forma de vida (nadadoras libres), la mayoría de especies marinas presentan distribución cosmopolita y se localizan en mares de todo el mundo (Ponce \& López, 2013). En las últimas décadas ha habido un gran interés sobre el grupo, debido a los crecientes problemas que generan proliferaciones masivas de estos organismos, conocidas como blooms. Estos episodios causan efectos disruptivos en las comunidades biológicas de la columna de agua (Mills, 2001; Purcell, et al., 2007; Boero, et al., 2008; Boero, 2013), y también afectan de manera negativa la economía y el equilibrio bioecológico de una región, especialmente sectores como el turismo por la disminución de bañistas, las pesquerías, debido a la ingestión de huevos y larvas de especies comerciales, las faenas de pesca, pues disminuye la abundancia de las capturas y se enredan en las artes de pesca, y, por último, porque causan la disminución de la biomasa planctónica disponible para las siguientes tramas ecológicas (Mills, 2001; Purcell, et al., 2007; Boero, et al., 2008; Boero, 2013). Aún no se conocen con precisión las causas de tales florecimientos, y aunque no hay consenso, se han sugerido el calentamiento global, las perturbaciones antropogénicas y la eliminación de los grandes depredadores en la red trófica (Mills, 2001; Purcell, et al., 2007; Boero, et al., 2008; Boero, 2013).

En las playas costeras, que son áreas de esparcimiento muy concurridas, estas proliferaciones son responsables de uno de los envenenamientos más comunes para el humano. Se conoce que más de 1.000 especies son tóxicas, y el contacto con ellas puede originar inmediatamente ronchas, vesículas dolorosas y pruriginosas con sensación urente, además de eritema y edema cutáneos, que rara vez llegan a la necrosis y el colapso cardiorrespiratorio (Auerbach, 1991; Vera, et al., 2004; Otero, 2012; Jouiaei, et al., 2015). Cuando suceden estas proliferaciones, las autoridades costeras tienden a restringir el acceso a las playas para evitar cualquier incidente (Boero, 2013).

En el Caribe colombiano hasta ahora el grupo más estudiado es el de los cnidarios que producen esqueletos carbonatados y son generalmente sésiles, en tanto que aún hay muchos vacíos de información sobre la taxonomía, la ecología y la distribución de los medusozoos. Hasta el momento se han registrado allí 16 especies de sifonóforos
(Hydrozoa) (Domínguez, 2002), siete de Scyphozoa (Moncaleano \& Niño, 1979; Cedeño-Posso, 2010) y dos de Cubozoa (Cedeño-Posso, 2010; Cedeño-Posso \& Lecompte, 2013a), mientras que en el Mar Caribe se han registrado 114 especies de la primera de estas clases, 12 de la segunda y cuatro de la tercera (Mianzan \& Cornelius, 1999; Humann \& Deloach, 2002). A nivel mundial el número aumenta significativamente a 3.678 especies de Hydrozoa, 191 de Scyphozoa y 47 de Cubozoa (WoRMS, 2017).

El objetivo de este trabajo fue aportar información sobre el grupo en el departamento del Atlántico, determinando, en lo posible, la diversidad estacionaria de las formas medusoides, con énfasis en el área turística de Puerto Velero. Dicha información es clave desde el punto de vista de la conservación, así como del manejo y el uso de las playas para las actividades turísticas.

\section{Materiales y métodos}

Área de estudio. La playa de Puerto Velero (1056 48,7'N, $75^{\circ} 02^{`} 12,8^{\prime}$ 'O) está ubicada al oeste de la playa de Caño Dulce y Puerto Caimán (municipio de Tubará, Atlántico) sobre la costa Caribe de Colombia (Figura 1). En el sector de Puerto Velero se encuentra el parque Caribe Aqualand (1056’49,31'N, 75²'13,16”O) (Estación M1, Figura 1), en donde se concentraron las actividades de observación y de registro durante el estudio. Las otras localidades incluidas en el estudio, y sus respectivas estaciones, se presentan en la Figura 1.

Para el departamento del Atlántico, el promedio anual de la temperatura superficial del agua del mar oscila entre $\operatorname{los} 25,5^{\circ} \mathrm{C}$ y $29,5^{\circ} \mathrm{C}$ y la salinidad entre los 34,5 y los 37 ppm (Posada \& Henao, 2008). Hay dos períodos de lluvia (abril a mayo y octubre a noviembre) y dos períodos secos (diciembre a marzo y julio a septiembre) (Rangel-Buitrago, et al., 2017). Los vientos presentan valores medios de velocidad inferiores a 23 nudos. Los valores de velocidad más altos están asociados con los vientos que soplan desde el noreste durante el período seco, en tanto que los valores son más bajos entre septiembre y noviembre cuando los vientos soplan desde el este (Anfuso, et al., 2015).

La influencia del río Magdalena en las costas del departamento es evidente, debido al aporte de sedimentos y de materiales orgánicos e inorgánicos (Rangel-Buitrago, et al., 2017).

Fase de recolección y análisis. Los avistamientos y recolecciones en Puerto Velero se realizaron durante 17 meses, entre agosto del 2016 y diciembre del 2017, con visitas cada 15 días, aproximadamente. La estación comprendía $\sim 50.000 \mathrm{~m}^{2}$ y abarcaba desde la orilla de la playa hasta $200 \mathrm{~m}$ mar adentro (Estación M1, Figura 1). Se hicieron muestreos exploratorios de medusas mediante 'careteo' y nado libre limitados a la parte superficial de la columna de agua.

Los individuos recolectados se fijaron y preservaron en una solución de formalina entre 4 a $10 \%$ en agua de mar. La velocidad del viento y la altura de la ola se consultaron en la base de datos de Windguru (2017). 


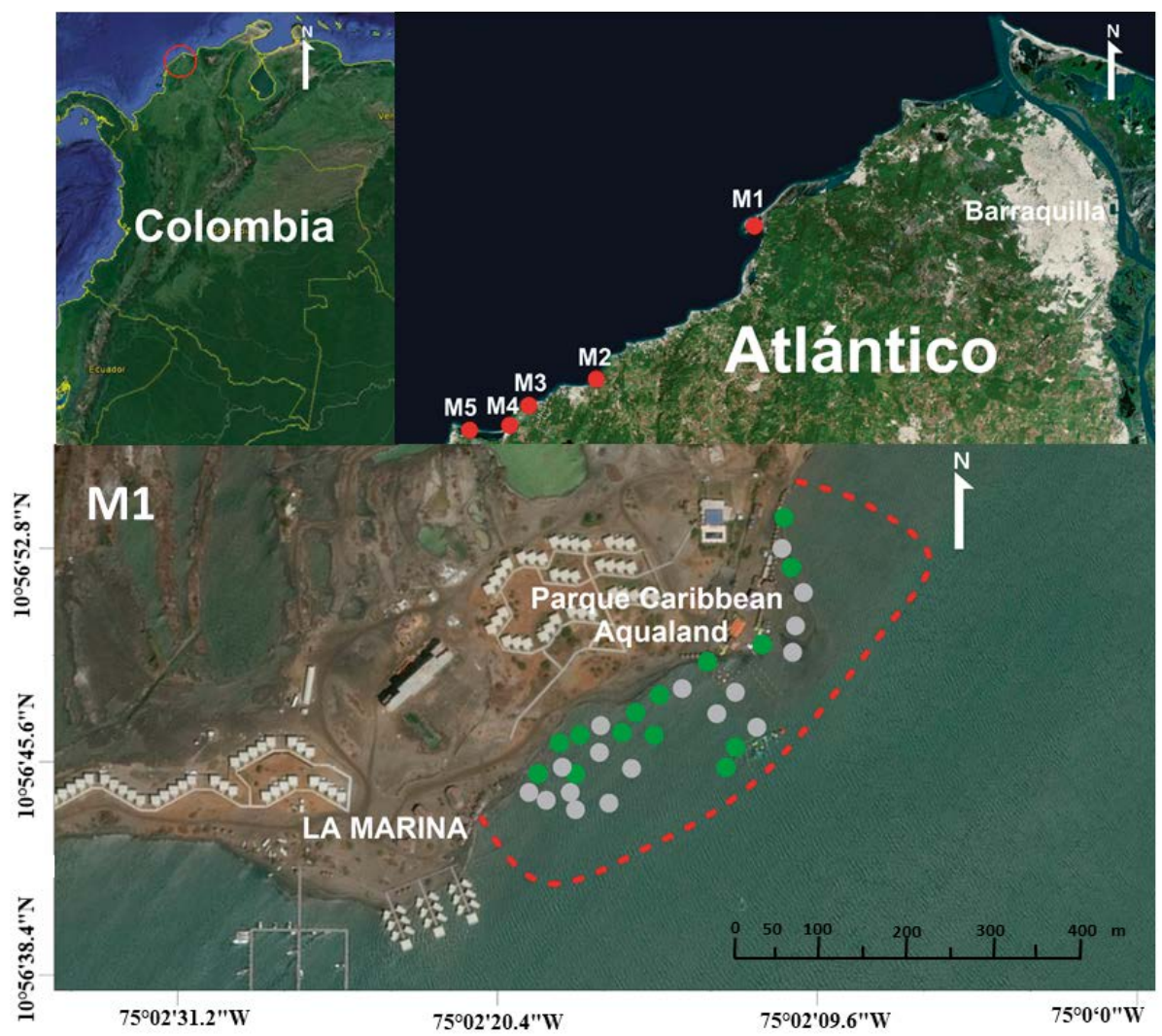

Figura 1. Área de estudio en el departamento del Atlántico. M1: Puerto Velero; M2: Aguamarina; M3: Bocatocinos; M4: Punta Astilleros; M5: Salinas de Galerazamba. Puntos grises: avistamientos (AVIS); puntos verdes: recolecciones (COL)

La identificación de los individuos se hizo en el Laboratorio de Zoología de la Universidad del Atlántico con ayuda de microscopios estéreo ZEISS Stemi 305; se utilizaron claves dicotómicas y pictóricas (Kramp, 1961; Hummelinck, 1968; Mianzan \& Cornelius, 1999). El material se incorporó en la colección biológica de la Universidad del Atlántico, sede del km 7, vía Puerto Colombia.

\section{Resultados}

En Puerto Velero se hicieron 39 visitas, en 21 de las cuales se avistaron medusas en nueve eventos: marzo, agosto y octubre de 2016, y abril, julio, agosto, septiembre, noviembre y diciembre de 2017. En las otras localidades (estaciones M2, M3, M4, M5), se obtuvieron registros de especies durante visitas puntuales. En la Tabla 1 se detallan las observaciones en campo y las recolecciones.

Se registraron 506 ejemplares (30 recolecciones y 476 avistamientos) pertenecientes a tres clases, cuatro órdenes y siete especies, distribuidos de la siguiente manera: para la clase Scyphozoa se registraron 104 ejemplares de la especie Chrysaora sp. (13 recolecciones y 91 avistamientos); 16 ejemplares de Cassiopea xamachana (6 recolecciones y 10 avistamientos); 305 ejemplares de Aurelia aurita (3 recolecciones y 302 avistamientos); 66 ejemplares de Lychnorhiza cf. lucerna (4 recolecciones y 62 avistamientos) y 10 ejemplares de Stomolophus meleagris (1 recolección y
9 avistamientos). En dos avistamientos se registró la especie Physalia physalis de la clase Hydrozoa, y, por último, se recolectaron tres ejemplares de Chiropsalmus quadrumanus de la clase Cubozoa (Tabla 1).

Se destacó la observación de más de 65 individuos de Chrysaora sp. y 238 individuos de A. aurita en una misma faena de pesca llevada a cabo el 4 de junio de 2017 en el sector de Puerto Velero.

A continuación se presenta la síntesis de las especies observadas. El ordenamiento taxonómico se hizo según el registro de WoRMS (2017):

Filo: Cnidaria Verrill, 1865

Clase: Hydrozoa Owen, 1843

Subclase: Hydroidolina Collins, 2000

Orden: Siphonophorae Eschscholtz, 1829

Suborden: Cystonectae Haeckel, 1887

Familia: Physaliidae Brandt, 1835

Género: Physalia Lamarck, 1801

Physalia physalis (Linnaeus, 1758) (Figura 2A)

Material observado en campo: dos ejemplares varados en la playa (marzo 15 de 2016), estación M3

Nueva localidad: Bocatocinos (Atlántico)

Distribución: cosmopolita (Yanagihara, et al., 2002;

Pontin \& Cruickshank, 2012; Haddad, et al., 2013). En el Caribe colombiano se ha registrado en Bolívar (Domínguez, 2002) 
Tabla 1. Registro de las recolecciones y avistamientos de medusas en las localidades de Puerto Velero, Aguamarina, Bocatocinos, Punta Astilleros y Salinas de Galerazamba en el departamento del Atlántico

\begin{tabular}{|c|c|c|c|c|c|c|c|c|c|c|c|c|c|c|c|c|c|c|c|}
\hline \multirow[t]{3}{*}{ Clase } & \multirow[t]{3}{*}{ Especie } & \multicolumn{6}{|c|}{2016} & \multicolumn{12}{|c|}{2017} \\
\hline & & \multicolumn{2}{|c|}{ Marzo } & \multicolumn{2}{|c|}{ Agosto } & \multicolumn{2}{|c|}{ Octubre } & \multicolumn{2}{|c|}{ Abril } & \multicolumn{2}{|c|}{ Julio } & \multicolumn{2}{|c|}{ Agosto } & \multicolumn{2}{|c|}{ Septiembre } & \multicolumn{2}{|c|}{ Noviembre } & \multicolumn{2}{|c|}{ Diciembre } \\
\hline & & AVIS & REC & AVIS & REC & AVIS & REC & AVIS & REC & AVIS & S REC & AVIS & REC & AVIS & REC & AVIS & REC & AVIS & REC \\
\hline Hidrozoa & $\begin{array}{l}\text { Physalia } \\
\text { physalis }\end{array}$ & $2^{\mathrm{C}}$ & 0 & 0 & 0 & 0 & 0 & 0 & 0 & 0 & 0 & 0 & 0 & 0 & 0 & 0 & 0 & 0 & 0 \\
\hline \multirow[t]{5}{*}{ Scyphozoa } & $\begin{array}{l}\text { Lychnorhiza } \\
\text { cf. lucerna }\end{array}$ & 0 & 0 & 0 & 0 & $32^{\mathrm{A}}$ & 0 & 0 & 0 & $1^{\mathrm{A}}$ & $1^{\mathrm{A}}$ & $\begin{array}{c}25^{\mathrm{A}} \\
4^{\mathrm{D}}\end{array}$ & $3^{\text {B }}$ & 0 & 0 & 0 & 0 & 0 & 0 \\
\hline & $\begin{array}{l}\text { Stomolophus } \\
\text { meleagris }\end{array}$ & 0 & 0 & 0 & 0 & $7^{\mathrm{A}}$ & 0 & 0 & 0 & 0 & $1^{\mathrm{A}}$ & $2^{\mathrm{A}}$ & 0 & 0 & 0 & 0 & 0 & 0 & 0 \\
\hline & Chrysaora sp. & 0 & 0 & 0 & 0 & 0 & 0 & $10^{\mathrm{A}}$ & $1^{\mathrm{A}}$ & $81^{\mathrm{A}}$ & $12^{\mathrm{A}}$ & 0 & 0 & 0 & 0 & 0 & 0 & 0 & 0 \\
\hline & Aurelia aurita & 0 & 0 & $25^{\mathrm{A}}$ & 0 & $15^{\mathrm{A}}$ & 0 & 0 & 0 & $243^{A}$ & $3^{\mathrm{A}}$ & 0 & 0 & $9^{\mathrm{A}}$ & 0 & $10^{\mathrm{A}}$ & 0 & 0 & 0 \\
\hline & $\begin{array}{l}\text { Cassiopea } \\
\text { xamachana }\end{array}$ & 0 & 0 & 0 & 0 & 0 & 0 & 0 & 0 & 0 & 0 & 0 & 0 & 0 & 0 & 0 & 0 & $10^{\mathrm{E}}$ & $6^{\mathrm{E}}$ \\
\hline Cubozoa & $\begin{array}{l}\text { Chiropsalmus } \\
\text { quadrumanus }\end{array}$ & 0 & 0 & 0 & 0 & 0 & 0 & 0 & 0 & 0 & 0 & 0 & $3^{\text {B }}$ & 0 & 0 & 0 & 0 & 0 & 0 \\
\hline
\end{tabular}

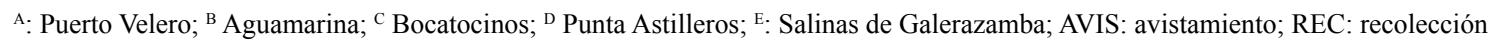

Comentario: la observación estuvo a cargo de estudiantes del programa de Biología de la Universidad del Atlántico durante una salida de campo hacia el sector de Bocatocinos.

Clase: Scyphozoa Goette, 1887

Subclase: Discomedusae Haeckel, 1880

Orden: Rhizostomeae Cuvier, 1799

Familia: Lychnorhizidae Haeckel, 1880

Género: Lychnorhiza Haeckel, 1880

Lychnorhiza cf. lucerna Haeckel, 1880 (Figura 2B)

Material examinado: un ejemplar (UARC-CNI 033), recolectado en la parte superficial de la columna de agua en el parque Caribe Aqualand, estación M1 (julio 9 de 2017); tres ejemplares (UARC-CNI 035, UARC-CNI 036 y UARCCNI 037) extraídos en redes durante actividades de pesca en el sector de la estación M2 (agosto 1 de 2017)

Material observado en campo: 32 ejemplares varados en la playa cerca de la estación M1 (octubre 12 de 2016) $\left(10^{\circ} 57^{\prime} 07,1^{\prime \prime} \mathrm{N}, 75^{\circ} 01^{\prime} 58,8^{\prime \prime} \mathrm{O}\right)$; un ejemplar nadando cerca del parque acuático de Puerto Velero (julio 19 de 2017) $\left(10^{\circ} 56^{\prime} 48,6^{\prime \prime} \mathrm{N}, 75^{\circ} 02^{\prime} 12,9^{\prime} \mathrm{O}\right)$; cuatro ejemplares varados en la playa en la estación M4 (agosto 1 de 2017); 21 ejemplares varados cerca de los límites de la estación M1 (agosto 20 de 2017) $\left(10^{\circ} 56^{\prime} 52,7^{\prime}{ }^{\prime} \mathrm{N}, 75^{\circ} 02^{\prime} 10,8^{\prime \prime} \mathrm{O}\right)$

Nueva localidad: Puerto Velero, Aguamarina y Punta Astilleros (Atlántico)

Distribución: costa atlántica de Suramérica. En el Caribe colombiano se ha registrada en Bolívar, Magdalena y La Guajira (Cedeño-Posso, 2010)

Características morfológicas: umbrela hemisférica con un promedio de $73 \pm 6,8 \mathrm{~mm}$ de diámetro $(\mathrm{n}=4)$; cuatro pares de brazos orales de tres alas de longitud de $52 \pm 12,8$ $\mathrm{mm}$ en promedio; gránulos de tamaño minúsculo, casi imperceptibles, en la exumbrela; coloración blanquecina tanto en la umbrela como en los brazos orales y ocho manchas de color azulmorado, dos en cada punto, redondeando el estómago; ocho ropalios incrustados en nichos profundos formando surcos sobre la exumbrela, cada uno de ellos protegido por dos lóbulos ropaliares pequeños, paralelos y punteados; 35 a 40 lóbulos marginales triangulares con puntas redondeadas; ausencia de la boca central y presencia de cuatro ostios genitales

Comentarios: el material recolectado correspondía a dos ejemplares juveniles y dos adultos, con algunas características concordantes con las de la especie Lychnorhiza lucerna, pero sin filamentos en los brazos orales ni la coloración azul en el margen umbrelar, por lo cual en este estudio se registró como Lychnorhiza cf. lucerna, según la propuesta de CedeñoPosso \& Lecompte (2013b). La presencia de ejemplares varados en la playa en octubre del 2016 probablemente se asocia con los vientos moderados (11,3 nudos) y con el oleaje (1,27 m) en ese sector (Windguru, 2017).

Según diversos estudios, la descripción inicial de la especie Lychnorhiza lucerna presentaba un gran número de sinonimias, lo cual ha llevado a proponer revisiones (Cedeño-Posso \& Lecompte, 2013b). Los ejemplares encontrados en la costa Caribe presentaban una variedad morfológica diferente a las reportadas en otros lugares del mundo, por lo que sería de gran importancia llevar a cabo análisis moleculares que permitan confirmar si se trata de otra especie o corresponde a una variación morfológica producto de las condiciones ambientales del lugar (CedeñoPosso \& Lecompte, 2013b).

Familia: Stomolophidae Haeckel, 1880

Género: Stomolophus Agassiz, 1862 
Stomolophus meleagris Agassiz, 1862 (Figura 2C)

Material examinado: un ejemplar (UARC-CNI 034) recolectado en la parte superficial de la columna de agua en el parque Caribe Aqualand, estación M1 (julio 16 de 2017)

Material observado en campo: siete ejemplares varados cerca de los límites de la estación M1 (octubre 12 de 2016) $\left(10^{\circ} 57^{\prime} 03,0^{\prime \prime} \mathrm{N}, 75^{\circ} 01^{\prime} 43,7^{\prime \prime} \mathrm{O}\right)$ y dos avistamientos en la parte superficial de la columna de agua en la estación M1 (agosto 20 de 2017)

Nueva localidad: Puerto Velero (Atlántico)

Distribución: mar Caribe, golfo de México, Caribe colombiano (Bolívar y Magdalena), mar Mediterráneo, Atlántico norte y sur, océano Pacífico del norte (Cedeño-Posso, et al., 2016)

Características morfológicas: umbrela hemisférica de $43 \mathrm{~mm}$ de diámetro $(\mathrm{n}=1)$; superficie exumbrelar lisa; ocho brazos orales dicotómicos, cortos y fusionados formando un pseudo-manubrio sin filamentos y con una boca central primaria; dos escapulas (16 en total) con aperturas bucales en la base de cada brazo oral de $15 \mathrm{~mm}$ de longitud, y ocho ropalios

Comentarios: el material recolectado correspondía a un ejemplar juvenil. Al igual que en el caso de Lychnorhiza cf. lucerna, se considera que los ejemplares varados en la playa pudieron estar asociados con los vientos moderados $(11,3$ nudos $)$ y el oleaje $(1,27 \mathrm{~m})$ que se presentaban en el sector.

Familia: Cassiopeidae Agassiz, 1862

Género: Cassiopea Péron \& Lesueur, 1810

Cassiopea xamachana (Fig. 2D-E)

Material examinado: en total seis ejemplares de la misma localidad. Catalogados de la siguiente manera: dos ejemplares UARC-CNI 041, dos ejemplares UARC-CNI 042, un ejemplar UARC-CNI 043 y un ejemplar UARC-CNI 044. Los ejemplares se recolectaron en el borde de una laguna temporal ubicada en la parte posterior de la playa de Salinas de Galerazamba, estación M5 (diciembre 17 de 2017)

Material observado en campo: diez ejemplares observados en el mismo punto del material examinado

Nueva localidad: Salinas de Galerazamba, Atlántico, Bolívar

Distribución: se encuentra en todo el Mar Caribe desde el extremo sur de Florida (Hummelinck, 1968; Fitt \& Costley, 1998). En el Caribe colombiano se ha registrado en Magdalena (Cedeño-Posso, 2010)

Características morfológicas: umbrela achatada y cóncava en la zona aboral con un promedio de 64,2 $\pm 10,2 \mathrm{~mm}$ de diámetro $(n=6)$; entre 72 y 86 lóbulos marginales pequeños y redondos, separados entre sí por surcos profundos; ocho brazos orales que exceden el margen umbrelar, 30,3 $\pm 11,9$ $\mathrm{mm}$ de largo en promedio, con ramificaciones irregulares provistas de aperturas diminutas de bocas secundarias rodeadas de un collar de tejido; ausencia de boca central y presencia de vesículas de 5 a $15 \mathrm{~mm}$ de largo en forma de cinta de coloración azul oscuro; 14 a 18 ropalios protegidos

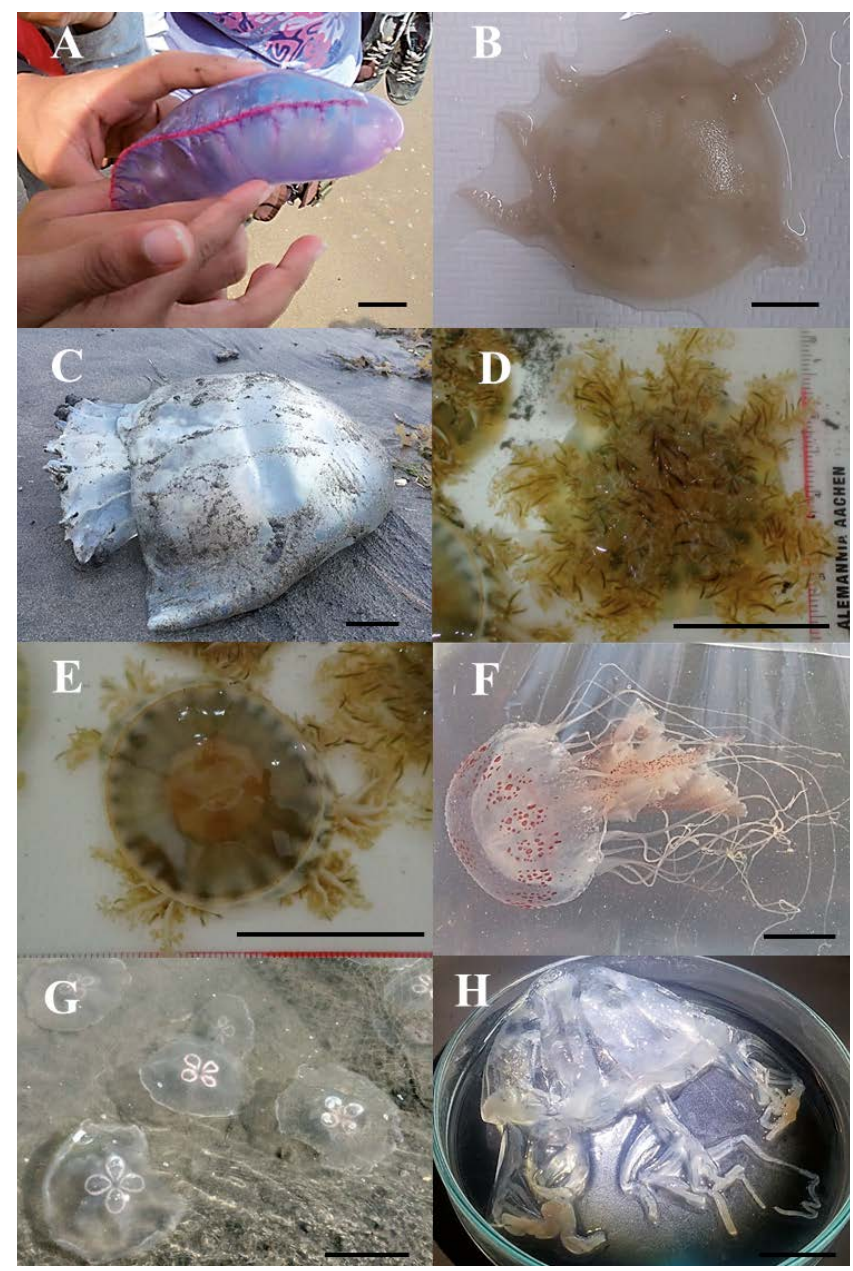

Figura 2. Registros fotográficos de las especies del subfilo Medusozoa presentes en la línea de costa del departamento del Atlántico. A. Physalia physalis; B. Lychnorhiza cf. lucerna; C. Stomolophus meleagris; Cassiopea xamachana: D. posición oral, E. posición aboral; F. Chrysaora sp.; G. Aurelia aurita; H. Chiropsalmus quadrumanus. Escalas: A-F, H: $2 \mathrm{~cm}, \mathrm{G}: 20 \mathrm{~cm}$

en nichos con una fina membrana; canales radiales que se comunican por anastomosis y generalmente divergen de los vasos radiales hacia la periferia formando un ángulo más o menos agudo; cuatro gónadas y cuatro fosas subgenitales separadas; coloración verdosa-azul debido a la simbiosis de numerosas zooxantelas densamente empaquetas

Comentarios: los ejemplares se encontraron en el borde del agua atrapados por material vegetal en una pequeña laguna formada en la parte posterior de la playa cuando todavía estaban vivos y flotaban. En el momento de la observación se presentaban vientos que oscilaban entre los 15 y 25 nudos (Windguru, 2017). Se presume que por estas condiciones fueron arrastrados desde la laguna anexa a la salina a esta parte de la playa. Las salinas de Galerazamba limitan con el departamento de Bolívar, lo que corresponde al registro de detección de estos organismos más al sur.

Orden: Semaeostomeae L. Agassiz, 1862 
Familia: Pelagiidae Gegenbaur, 1856

Género: Chrysaora Péron \& Lesueur, 1810

Chrysaora sp. (Fig. 2F)

Material examinado: un ejemplar (UARC-CNI 019), abril 10 de 2017, estación M1; dos ejemplares (UARC-CNI 020 y UARC-CNI 021), julio 2 de 2017, estación M1; nueve ejemplares (UARC-CNI 023, UARC-CNI 024, UARC-CNI 025, UARC-CNI 026, UARC-CNI 027, UARC-CNI 028, UARC-CNI 029, UARC-CNI 030 y UARC-CNI 031), julio 4 de 2017, estación M1; un ejemplar (UARC-CNI 032), julio 5 de 2017, estación M1

Material observado en campo: diez ejemplares nadando en la parte superficial de la columna de agua en la estación M1 (abril 11 de 2017); 65 ejemplares nadando en la parte superficial de la columna de agua en la estación M1 (julio 4 de 2017); un ejemplar varado en la orilla de la playa cerca de la estación M1 (julio 5 de 2017) $\left(10^{\circ} 56^{\prime} 47,3^{\prime \prime} \mathrm{N}\right.$, $75^{\circ} 02^{\prime} 17,1$ 'O); ocho ejemplares varados y seis nadando cerca de la estación M1 (julio 9 de 2017) $\left(10^{\circ} 57^{\prime} 2,99^{\prime} \mathrm{N}\right.$, $75^{\circ} 2$ '3,51'O), y un ejemplar varado en la playa cerca de la estación M1 (julio 23 de 2016) $\left(10^{\circ} 57^{\circ} 0,56^{\prime} \mathrm{N}\right.$, 75²'8,44'O)

Nueva localidad: Puerto Velero (Atlántico)

Distribución: Atlántico occidental, desde Jamaica hasta la costa norte de Argentina (Morandini, et al., 2006). En el Caribe colombiano se ha registrado en Magdalena (CedeñoPosso, 2010)

Características morfológicas: umbrela hemisférica de $61,54 \pm 17,73 \mathrm{~mm}$ de diámetro en promedio ( $\mathrm{n}=13$ ); exumbrela de color blanco con manchas de puntos rojizos; en los ejemplares se encontraron los cuatros patrones de coloración de la exumbrela registrados en la literatura; 24 tentáculos con una medida máxima de $40 \mathrm{~cm}$ de largo en algunos ejemplares, tres por cada octante; ocho ropalios sin ocelos, y cuatro brazos orales con los bordes festoneados, de 58,15 $\pm 26,3 \mathrm{~mm}$ de largo

Comentarios: los ejemplares recolectados de la especie Chrysaora sp. eran juveniles, por lo tanto, no habían desarrollado algunos caracteres morfológicos necesarios para definir la especie. Podrían tratarse de $C$. lactea o $C$. quinquecirrha, ambas especies registradas para el Caribe.

Vale la pena destacar que el 4 de julio de 2017, en la estación de muestreo M1 (1056’46,2”N, 7502'18,3”O), se encontró una acumulación de 65 individuos de Chrysaora sp. Para esta fecha se registró un frente frío con una velocidad del viento de 15 nudos en dirección suroeste. Además, algunos ejemplares estaban acompañados por peces juveniles de la familia Carangidae, que nadaban sobre la umbrela y alrededor de los brazos orales.

Familia: Ulmaridae Haeckel, 1879

Género: Aurelia Lamarck, 1816

Aurelia aurita Linnaeus, 1758 (Fig. 2G)

Material examinado: tres ejemplares (UARC-CNI 022) nadando en la estación M1 (julio 4 de 2017)
Material observado en campo: 25 ejemplares varados en la playa en la estación M1 (agosto 1 de 2016); 15 ejemplares nadando en la estación M1 (octubre 29 de 2016); 238 ejemplares nadando en la estación M1 (julio 4 de 2017); dos ejemplares varados en la playa en la estación M1 y tres flotando en la playa, sin señales de movimiento, cerca del parque Caribe Aqualand (julio 9 de 2017); dos ejemplares varados en la playa en la estación M1 (septiembre 9 de 2017); siete ejemplares nadando en la columna de agua superficial en la estación M1 (septiembre 10 de 2017).

Nueva localidad: Puerto Velero (Atlántico)

Distribución: cosmopolita (Dawson \& Jacobs, 2001; Schroth, et al., 2002). En el Caribe colombiano se ha registrado en Magdalena, Bolívar y La Guajira (CedeñoPosso, 2010)

Características morfológicas: umbrela hemisférica con $301 \pm 80,8 \mathrm{~mm}$ de diámetro en promedio $(\mathrm{n}=14)$; campana plana con forma de cúpula, firme y transparente; cuatro anillos de gónadas de color azulado-rosa con largo y ancho promedio $(54,44 \pm 54,6$ y $40,73 \pm 13,4 \mathrm{~mm}$, respectivamente), y cuatro brazos orales de 136,82 $\pm 42,1 \mathrm{~mm}$ de longitud en promedio

Comentarios: los ejemplares recolectados eran adultos y exhibían todas las características típicas de la especie. El 4 de julio de 2017 se encontró en la estación M1 una acumulación de $A$. aurita que coincidió con un frente frío registrado en el sector (velocidad de 15 nudos, dirección suroeste). Los 238 ejemplares registrados como material observado en campo fueron encontrados en una red de pesca de más de $40 \mathrm{~m}$ de largo por pescadores que estaban en la zona.

Clase: Cubozoa Werner, 1973

Orden: Chirodropida Haeckel, 1880

Familia: Chiropsalmidae Thiel, 1936

Género: Chiropsalmus Agassiz, 1862

Chiropsalmus quadrumanus (F. Muller, 1859) (Figura 2H)

Material examinado: tres ejemplares (UARC-CNI 038, UARC-CNI 039 y UARC-CNI 040), en redes de pesca, estación M2 (agosto 1 de 2017)

Nueva localidad: Playa Aguamarina (Atlántico)

Distribución: costa Atlántica de Sur América y mar Caribe (Kramp, 1961; Mianzan \& Cornelius, 1999). En el Caribe colombiano se ha registrado en Magdalena (CedeñoPosso, 2010; Cedeño-Posso \& Lecompte, 2013a)

Características morfológicas: forma cúbica, con un ancho de la campana de 59,3 $\pm 6,6$ y un largo de 39,6 \pm 2 $\mathrm{mm}$ en promedio $(\mathrm{n}=3)$; cuatro ropalios a cada lado de la campana dentro de los nichos ropaliares en forma de domo; cuatro pedalias ramificadas de $25 \pm 3,5 \mathrm{~mm}$ en promedio, con dos patrones en el desprendimiento de los tentáculos (55-5-5-5 y 6-6-6-6); manubrio con cuatro labios, y coloración blanca con tonalidad translúcida y blanquecina

Comentario: los individuos fueron recolectados por pescadores del sector quienes los encontraron enredados en la red de fondo. Los ejemplares correspondían a ejemplares 
juveniles de la especie (Dra. Ilka Straehler-Pohl, comunicación personal, febrero 1 de 2018) y, por lo tanto, no presentaban todas las características propias descritas para un adulto; los ejemplares juveniles presentan un tentáculo y llegan a tener nueve cuando alcanzan su tamaño corporal de adulto.

\section{Discusión}

Se hicieron nuevos registros para el departamento del Atlántico. Únicamente la especie $A$. aurita fue previamente registrada por la Corporación Autónoma Regional del Atlántico (C.R.A., 2016), probablemente porque su gran tamaño la hace más notoria $\mathrm{y}$, por ende, los avistamientos pueden ser más frecuentes que los de otras especies; sin embargo, ese registro anterior no contiene la ubicación ni la fecha del avistamiento de la especie.

Con relación a la metodología empleada, ésta se centró en la recolección y observación de organismos que llegan a la línea de la costa, por lo que posteriores estudios con diversas metodologías costa afuera (chinchorros playeros, redes para peces mesopelágicos e, incluso, redes de zooplacton e ictioplancton), seguramente ayudarán a establecer la presencia de un mayor número de especies.

No se pudieron establecer correlaciones entre la presencia de medusas y los parámetros ambientales medidos in situ por falta de información como la proveniente de boyas oceanográficas. En el futuro sería importante contar con dichas herramientas para establecer relaciones entre las variables ambientales y los pulsos de proliferación de estos organismos. Sin embargo, se observó que el aumento en la velocidad del viento puede estar relacionado con los varamientos de estos individuos en la playa turística de Puerto Velero y en otros sectores. Se evidenció que las especies $A$. aurita, S. meleagris y $L$. cf. lucerna frueron arrastradas hacia a la playa, donde muchas de ellas quedaron varadas debido a velocidades del viento entre los 7,55 y 15,12 nudos (Windguru, 2017) durante octubre de 2016. En julio de 2017, las especies $A$. aurita y Chrysaora sp., también se vieron afectadas por vientos con velocidades que oscilaban entre los 13,67 y 18,66 nudos (Windguru, 2017).

Los estudios de línea de base y el conocimiento de las especies que se pueden encontrar localmente son una herramienta de gestión que permite tomar medidas de manejo adecuadas en cualquier lugar. El sector de Puerto Velero se proyecta como un punto de desarrollo turístico en la región, y es visitado por una gran cantidad de personas que, infortunadamente, no reconocen a estos organismos como habitantes naturales del entorno y, en muchos casos, los extraen y los dejan en la playa sin razón. Estas acciones pueden afectar la cadena trófica del ecosistema, causando un efecto dominó en otros organismos de interés comercial, como los peces, u otros que puedan ser sus depredadores naturales.

La información presentada constituye la línea de base para un mejor conocimiento y manejo de los recursos y la biodiversidad marina, así como de la gestión en playas con vocación turística, y permite implementar planes conjuntamente con la comunidad local y los visitantes del sector.

\section{Conclusiones}

Este estudio aporta al conocimiento de la composición de las especies del subfilo Medusozoa encontradas en algunas playas turísticas del departamento del Atlántico. A pesar de que no se consideraron los parámetros ambientales locales para determinar su variación estacional, se pudo observar dónde y cuándo se presentaron proliferaciones masivas de algunas especies. En la medida en que se visiten otras localidades y se utilicen otras herramientas de observación, se espera aumentar el número de registros de especies en este sector del Caribe colombiano.

\section{Agradecimientos}

Al Grupo de Investigación en Geología, Geofísica y Procesos Marino-Costeros (GGPMC) de la Universidad del Atlántico; a la Universidad del Atlántico por la disponibilidad de los laboratorios y el uso de los estereoscopios; a José Moreno y Martha Torres por facilitarnos la instalaciones en el parque Caribe Aqualand; a Carlos Altamar por suministrarnos la fotografía de la Figura 2A y el reporte de $P$. physalis en Bocatocinos (Atlántico); a José Bustamante por la fotografía de la Figura $2 \mathrm{C}$ y el reporte de avistamientos de medusas; a Andre Morandini e Ilka Straehler-Pohl, por la confirmación de la cubomedusa; a la comunidad de pescadores en Puerto Velero y Aguamarina (Atlántico), y al grupo de estudiantes del GGPMC por su gran colaboración.

\section{Conflicto de intereses}

Los autores declaramos no tener conflicto de intereses.

\section{Contribución de los autores}

Jeferson Durán-Fuentes: actividades de campo, identificación de las muestras, escritura y análisis de la información; Adriana Gracia C.: financiación del proyecto, actividades de campo, escritura y análisis de la información; Carlos Osorio M.: actividades de campo y escritura de la información; Cristina Cedeño-Posso: escritura y análisis de la información.

\section{Referencias}

Anfuso, G., Rangel-Buitrago, N., Correa, I. (2015). Evolution of sands pits along the Caribbean Coast of Colombia: Natural and human influences. En: Randazzo, G., Jackson, D., Cooper, J.A. (Eds.), Sand and Gravel Spits. Springer, Nueva York, pp. 1-21. https://doi.org/10.1007/978-3-31913716-2

Auerbach, P. (1991). Marine Envenomations. New England Journal of Medicine, 324: 1332-1338. https://doi.org/10. 1056/NEJM199108153250707

Boero, F. (2013). Review of jellyfish blooms in the Mediterranean and Black Sea. Studies and Reviews. General Fisheries Commission for the Mediterranean. Rome, Italia. FAO. https://doi.org/10.1080/17451000.2014.880790 
Boero, F., Bouillon, J., Gravili, C., Miglietta, M.P., Parsons, T., Piraino, S. (2008). Gelatinous plankton: Irregularities rule the world (sometimes). Marine Ecology Progress Series. 356: 299310 . https://doi.org/10.3354/meps07368

Corporación Autónoma Regional del Atlántico - C.R.A. (2016). Atlas de Flora y Fauna Marino Costero del departamento del Atlántico. Barranquilla, Colombia. Editorial Gente Nueva. p. 282.

Cedeño Posso, C. M. (2010). Composición preliminar de las medusas (Cnidaria: Cubozoa y Scyphozoa) de las aguas superficiales costeras de la región de Santa Marta. Tesis de pregrado, Universidad de Bogotá Jorge Tadeo Lozano. https://doi.org/10.13140/RG.2.1.4327.9840

Cedeño-Posso, C. \& Lecompte, O. (2013a). Cubomedusas (Cnidaria: Cubozoa) del mar Caribe colombiano. Acta Biológica Colombiana. 18: 205-210.

Cedeño-Posso, C. \& Lecompte, O. (2013b). Primer registro de medusas del género Lychnorhiza (Cnidaria: Scyphozoa: Rhizostomae: Lychnorhizidae) en el Mar Caribe Colombiano. Biol. Invest. Mar. Cost. 42 (2): 387-393.

Cedeño-Posso, C.M., Suaréz-Mozo, N.Y., Castaño-Goméz, J. (2016). La medusa bala de cañón (Stomolophus meleagris) en Colombia, revisión de su distribución y primer reporte en el océano Pacífico. Revista Biodiversidad Neotropical. 6: $221-226$.

Collins, A.G. (2002). Phylogeny of medusozoa and the evolution of cnidarian life cycles. Journal of Evolutionary Biology. 15: 418-432. https://doi.org/10.1046/j.1420-9101.2002.00403.x

Collins, A.G., Schuchert, P., Marques, A.C., Jankowski, T., Medina, M., Schierwater, B. (2006). Medusozoan phylogeny and character evolution clarified by new large and small subunit rDNA data and an assessment of the utility of phylogenetic mixture models. Systematic Biology. 55: $97-115$. https://doi.org/10.1080/10635150500433615

Daly, M., Brugler, M.R., Cartwright, P., Collins, A.G., Dawson, M.N.,Fautin, D.G., France, S.C., McFadden, C.S., Opresko, D.M., Rodríguez, E., Romano, S.L, Stake, J.L. (2007). The phylum Cnidaria: A review of phylogenetic patterns and diversity 300 years after Linnaeus. Zootaxa. 1668: $127-182$. https://doi.org/10.5281/zenodo.180149

Dawson, M., Jacobs, D. (2001). Molecular evidence for cryptic species of Aurelia aurita (Cnidaria, Scyphozoa). The Biological Bulletin. 200: 92-96. https://doi.org/10.2307/1543089

Domínguez, H. (2002). Hidromedusas y Sifonósforo (Cnidaria: Hydrozoa) de las aguas superficiales de la Bahía de Gaira, Caribe colombiano: taxonomía, abundancia y relación con la oferta alimenticia (Tesis de pregrado, Universidad de Bogotá Jorge Tadeo Lozano).

Fitt, W. K. \& Costley, K. (1998). The role of temperature in survival of the polyp stage of the tropical rhizostome jellyfish Cassiopea xamachana. Journal of Experimental Marine Biology and Ecology. 222: 79-91. https://doi.org/10.1016/ S0022-0981(97)00139-1

Gasca, R., Loman-Ramos, L. (2014). Biodiversidad de Medusozoa (Cubozoa, Scyphozoa e Hydrozoa) en México. Revista Mexicana de Biodiversidad. 85: 154-163. https:// doi.org/10.7550/rmb.32513

Haddad-Junior, V., Virga, R., Bechara, A., da Silveira, F. L., Morandini, A. C. (2013). An outbreak of Portuguese manof-war (Physalia physalis - Linnaeus, 1758) envenoming in Southeastern Brazil. Revista Da Sociedade Brasileira de Medicina Tropical. 46: 641-644. http://doi.org/10.1590/00378682-1518-2013

Humann, P. \& Deloach, N. (2002). Reef Creature Identification: Florida, Caribbean, Bahamas. The Reef Set (2nd ed.). Jacksonville, Florida USA: New Word Publications, Inc.

Hummelinck, P. (1968). Caribbean scyphomedusae of the genus Cassiopea. Studies of fauna of Curaçao and other Caribbean Islands. 23: 1131-1143.

Jouiaei, M., Yanagihara, A.A., Madio, B., Nevalainen, T.J., Alewood, P.F., Fry, B.G. (2015). Ancient venom systems: A review on Cnidaria toxins. Toxins. 7: 2251-2271. https:// doi.org/10.3390/toxins 7062251

Kramp, P.L. (1961). Synopsis of the medusae of the world. Journal of the Marine Biological Association of the UK. 40: 7-382.

Marques, A.C., Collins, A.G. (2004). Cladistic analysis of Medusozoa and cnidarian evolution. Invertebrate Biology. 123: 23-42. https://doi.org/10.1111/j.1744-7410.2004.tb00139.x

Mianzan, H. \& Cornelius, P. (1999). Cubomedusae and Scyphomedusae. South Atlantic Zooplankton. 1: 513-559.

Mills, C.E. (2001). Jellyfish blooms: Are populations increasing globally in response to changing ocean conditions? Hydrobiologia. 451: 55-68.

Moncaleano, A. \& Niño, L. (1979). Celenterados planctónicos de la Bahía de Cartagena, descripciones y notas ecológicas. Boletín Museo del Mar. 9: 37-96.

Morandini, A.C., Da Silveira, F.L., Cornelius, P.F. (2006). Redescription of Chrysaora lactea Eschscholtz, 1829 (Cnidaria, Scyphozoa) from the Brazilian coast, with designation of a neotype. Zootaxa. 1135: 29-48. https://doi. org/10.5281/zenodo. 171956

Otero, V. (2012). Dermatosis acuáticas. Revista de la Asociación Colombiana de Dermatología y Cirugía Dermatológica. 3: 281-284.

Ponce, D. P. \& López, E. (2013). Medusas: las bailarinas del mar. CONABIO Biodiversi. 109: 1-6.

Posada, B. \& Henao, W. (2008). Diagnóstico de la erosión en la zona costera del Caribe colombiano. Santa Marta, Colombia. INVEMAR, Serie Publicaciones Especiales No. 13: 34-35.

Pontin, D. R., Cruickshank, R. H. (2012). Molecular phylogenetics of the genus Physalia (Cnidaria: Siphonophora) in New Zealand coastal waters reveals cryptic diversity. Hydrobiologia. 686: 91-105. https://doi.org/10.1007/s10750011-0994-8

Purcell, J.E., Uye, S.I., Lo, W.T. (2007). Anthropogenic causes of jellyfish blooms and their direct consequences for humans: A review. Marine Ecology Progress Series. 350: 153-174. https://doi.org/10.3354/meps07093

Rangel-Buitrago, N., Williams, A., Anfuso, G., Árias, M., Gracia, A. (2017). Magnitudes, sources, and management of beach litter along the Atlántico department coastline, Caribbean coast of Colombia. Ocean and Coastal Management. 138: 142-157. https://doi.org/10.1016/j.ocecoaman.2017.01.021

Schroth, W., Jarms, G., Streit, B., Schierwater, B. (2002). Speciation and phylogeography in the cosmopolitan marine moon jelly, Aurelia sp. BMC Evolutionary Biology. 2: 1-10. https://doi.org/10.1186/1471-2148-2-1

Technau, U., Genikhovich, G., Kraus, J.E.M. (2015). Cnidaria. En: Wanninger, A. (ed.). Evolutionary Developmental 
Biology of Invertebrates 1: Introduction, Non-Bilateria, Acoelomorpha, Xenoturbellida, Chaetognatha. SpringerVerlag Wien. https://doi.org/10.1007/978-3-7091-1862-7_6

Vera K, C., Kolbach M., Zegpi, M.S., Vera F., Lonza J. (2004). Picaduras de medusas: actualización. Revista Médica de Chile. 132: 233-241. https://doi.org/10.4067/S003498872004000200014

Windguru. (2017). The windguru.cz / windguru.com server. Fecha de consulta: octubre del 2016 a agosto del 2017. Disponible en: http://www.windguru.cz
WoRMS. (2017). The World Register of Marine Species aims to provide the most authoritative list of names of all marine species globally, ever published. Fecha de consulta: febrero y diciembre de 2017. Disponible en: http://www. marinespecies.org/

Yanagihara, A. A., Kuroiwa, J. M. Y., Oliver, L. M., Kunkel, D. D. (2002). The ultrastructure of nematocysts from the fishing tentacle of the Hawaiian bluebottle, Physalia utriculus (Cnidaria, Hydrozoa, Siphonophora). Hydrobiologia. 489: 139-150. https://doi.org/10.1023/A:1023272519668 